\title{
Die epigäische Spinnenzönose (Araneae) auf Schotter- bänken der Mittelgebirgsbäche und -flüsse im Rheinischen Schiefergebirge (Nordhessen)
}

\author{
Janna SMIT
}

\begin{abstract}
Spider community (Araneae) of gravel bars along streams in lower mountainous areas (Rheinisches Schiefergebirge, Northern Hesse, Germany). The spider communities on gravel bars along streams in lower mountainous areas of Northern Hesse were investigated. Eight streams differently altered by human impact were studied: four small streams without weirs and impoundments, two regulated small streams and two larger regulated streams. The sampling sites were located in one kilometer stretches of the upper, middle and lower reach of each small stream, respectively. In addition the gravel bars in several floodplain sections of larger streams were investigated. Altogether 592 quantitative samples of spiders were made. 7027 spiders (Araneae) comprising 77 species were encountered. Only two species (Oedothorax agrestis, Pirata knorri) make up more than 70 percent of all spiders captured. Erigone atra and Erigone dentipalpis were frequently found along all streams investigated. While Diplocephalus permixtus and Diplocephalus protuberans were only encountered along the small streams, Oedothorax retusus and Pardosa amentata were predominantly recorded on gravelbars of large streams. Diplocephalus protuberans was recorded for the first time in Hesse. The mean abundances (median) ranged between 4 and 28 individuals $/ \mathrm{m}^{2} .198$ individuals $/ \mathrm{m}^{2}$ have been the maximum of abundance.
\end{abstract}

Key words: Araneae, gravel bar, small streams, Hesse, Germany

\section{EINLEITUNG}

Im Vergleich zu den großen Umlagerungsstrecken der alpinen Gewässer (vgl. HERING 1995, PLACHTER 1993) sind die Schotterbänke an Mittelgebirgsbächen schon allein wegen ihres meist geringen Flächenausmaßes eher unscheinbar. Sie stellen an naturnahen Mittelgebirgsbächen jedoch einen verbreiteten Habitattyp dar (SMIT et al. i. Dr.).

Bisherwurde die epigäische FaunaaufSchotterflächen fastausschließlich an den alpinen Fließgewässern untersucht (BIGOT \& GAUTIER 1982, BOUMEZZOUGH 1983, BOUMEZZOUGH \& MUSSO 1983, HERING 1995, MANDERBACH \& REICH 1995, PLACHTER 1986). Detaillierte 
Arbeiten zur Spinnenfauna von alpinen Wildfüssen sind erst in jüngster Zeit erschienen(DRÖSCHMEISTER 1994, FRAMENAUetal. 1996, STEINBERGER i. Dr.). Nur selten sind Schotterufer außeralpiner, größerer Flüsse untersucht worden (BIGOT \& FAVET 1985, SIEPE 1985).

Die Spinnenfauna an schotterführenden Mittelgebirgsbächen ist bisher fast völlig unerforscht. Dies ist umso erstaunlicher, als daß auf Schotterbänken die Gruppe der echten Spinnen (Araneae) neben Laufkäfern und Springschwänzen zu den dominierenden Tiergruppen dieses Lebensraumes gehört (MANDERBACH \& REICH 1995, SMIT et al. i. Dr.).

Die vorliegende Arbeitentstand im Rahmen einer größeren Untersuchung zu Morphologie und Besiedlung von Schotterbänken entlang Mittelgebirgsbächen. Die Ergebnisse zurMorphologie wurden an anderer Stelle publiziert (SMIT et al. i. Dr.).

Folgende Fragestellungen standen im Mittelpunkt der Untersuchung:

- Verändert sich die Artenzusammensetzung der Spinnen auf Schotterbänken im Längsverlauf eines Fließgewässers?

- Wie wirken sich wasserbauliche Maßnahmen auf die Spinnenzönose und deren Individuendichten aus?

Untersuchungsraum und Untersuchungsgewässer

Es wurden Schotterbänke entlang von sechs Bächen und zwei kleineren Flüssen am Ostrand des Rheinischen Schiefergebirges in Nordhessen untersucht (Abb.1). Die untersuchten Fließgewässerabschnitte liegen auf Höhen zwischen 212 und 520 m ü.NN.

An den Mittelgebirgsbächen wurden jeweils $1 \mathrm{~km}$ lange Gewässerabschnitte im Epi-, Meta- und Hyporhithral untersucht. Die Bäche fließen durch Wiesentäler in Laubwaldgebieten, die über weite Strecken direkt an eine Gewässerseite heranreichen. Im weiteren Verlauf dieser Fließgewässer weiten sich die Wiesentäler auf, ein mehr oder weniger dichter Ufergehölzsaum ist vorhanden. Lediglich im Metarhithralbereich des Linspher Baches fehlen über größere Strecken Ufergehölze. Zahlreiche Schotterbänke sind an diesen anthropogen kaum beeinträchtigten Gewässerabschnitten anzutreffen. An den Flüssen Lahn und Eder wurden jeweils mehrere Untersuchungsabschnitte im Epipotamal ausgewählt.

Um die wasserbaulichen Maßnahmen an den Gewässern quantifizieren zu können, wurden alle Längs, Quer- und Sohlverbauungen in den Untersuchungsstrecken kartiert (vgl. SMIT et al. i. Dr.). Danach konnte der 


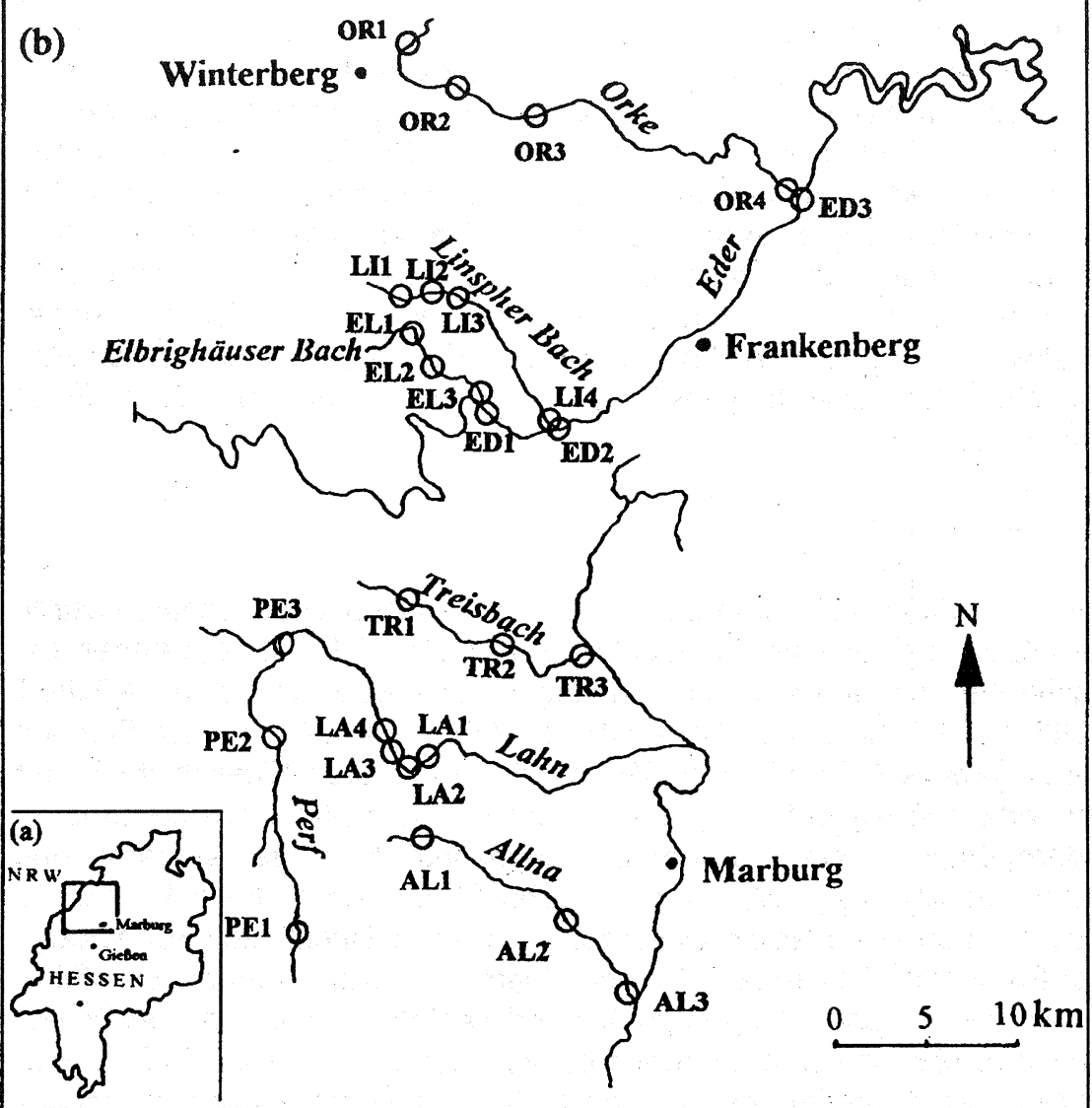

Abb. 1: Lage des Untersuchungsgebietes (a) und der $1 \mathrm{~km}$ langen Untersuchungsabschnittean den Fließgewässern in Nordhessen (b). Elbrighäuser Bach (EL1, EL2, EL3), Linspher Bach (LI1, LI2, LI3, LI4), Orke (OR1, OR2, OR3), Treisbach (TR1, TR2, TR3), Allna (AL1, AL2, AL3), Perf (PE1, PE2, PE3), Lahn (LA1, LA2, LA3) und Eder (ED1, ED2, ED3).

Elbrighäuser in ganzer Länge, Linspher Bach, Treisbach und Orke mit Ausnahme der Unterläufe als naturnah eingestuft werden. Sämtliche Untersuchungsabschnitte von Allna, Perf, Lahn und Eder sind wasserbaulich stark beeinträchtigt. Sie liegen meist im Einflußbereich von Ortschaften und fließen durch intensiv landwirtschaftlich genutzte Bereiche. An Lahn und Eder konnten sich lediglich hinter Wehren noch Kiesbänke ausbilden. 
Ein Großteil der Schotterbänke an den Mittelgebirgsbächen ist nicht größer als $9 \mathrm{~m}^{2}$ (SMIT et al. i. Dr.). An naturnahen Bächen im Rheinischen Schiefergebirge beträgt die durchschnittliche Anzahl 52 Schotterbänke pro Kilometer Gewässer, während sich an den verbauten Abschnitten lediglich durchschnittlich 7 Kiesbänke pro Kilometer befinden (SMIT et al. i. Dr.). Aufknapp $80 \%$ der kartierten Kiesbänke an den Mittelgebirgsbächen weist das Substrat eine Korngröße von bis zu $10 \mathrm{~cm}$ auf. Im Mai betrug die Vegetationsbedeckung auf fast allen Kiesbänken unter $5 \%$ der Fläche. Ein Großteil der Kiesbänke in den Epirhithralabschnitten wurde im Laufe des Jahres miteiner Pestwurz-Flur bedeckt. An Lahn und Ederwurde häufig das Rohrgrasglanzröhricht und später im Jahr das Drüsige Springkraut (Impatiens glandulifera) angetroffen.

\section{METHODEN}

Die Uferfauna der Schotterbänke in den Untersuchungsabschnitten wurde jeweils zu drei verschiedenen Jahreszeiten (Mai, Juli und September) mit flächenbezogenen Handaufsammlungen aufgenommen (vgl. ALMQUIST 1969, 1973, MANDERBACH \& REICH 1995). Die besammelten Flächen waren jeweils $0,57 \mathrm{~m}^{2}$ groß. Während die obersten Kiesschichten der Probeflächen vorsichtig abgetragen wurde, wurden sämtliche erkennbaren Arthropoden mit einem Exhaustor eingesaugt. Nach der Aufsammlung wurde die Fläche mit Wasser überschüttet, um Tiere, die sich tiefer im Substrat aufhielten, an die Oberfläche zu treiben. Direkt nach Beendigung der Probennahme wurden sämtliche Tiere in 70 \%iges Ethanol überführt.

Bei den Aufsammlungen im Juli und September wurde an den Fließgewässerabschnitten mit Schotterbankdichten von mehr als 40 Schotterbänken pro Kilometer jeweils jede fünfte Schotterbank beprobt, bei der Aufsammlung im Mai nur jede zehnte. An Untersuchungsabschnitten mit sehr geringer Schotterbankdichte wurden jeweils auf allen Schotterbänken Proben genommen. Insgesamtlagen 592 flächenbezogene Handaufsammlungen für vergleichende Auswertungen vor, von denen 531 direkt an der Wasserlinie und 61 in mehr als $1 \mathrm{~m}$ Entfernung von der Wasserlinie genommen wurden.

Die Bestimmung der Spinnen erfolgte vornehmlich mit den Bestimmungsschlüsseln von ROBERTS (1993 a,b,c), HEIMER \& NENTWIG (1991) und RUZICKA (1978). Die Nomenklatur richtet sich nach PLATNICK (1993).

Anhand von Literaturdaten (HEIMER \& NENTWIG 1991, MAURER \& HÄNGGI 1990) wurden die nachgewiesenen Spinnenarten in ökologische Gruppen eingeteilt und hierdurch eine Gruppe stenotoper Uferarten identifiziert. 


\section{ERGEBNISSE}

Mit flächenbezogenen Handaufsammlungen ( 592 Proben à 0,57 $\mathrm{m}^{2}$ ) wurden insgesamt 7027 Spinnen gefangen, von denen $51 \%$ adult, $17 \%$ im subadulten und $32 \%$ im juvenilen Stadium waren. Sie konnten 77 Spinnenarten aus 13 Familien zugeordnet werden (s. Tab. 1).

$58 \%$ aller Arten gehören der Familie der Baldachinspinnen (Linyphiidae) an. Die Wolfspinnen (Lycosidae) sind mit knapp $13 \%$ der vorgefundenen Arten vertreten. Die Verteilung der Individuen aufdie Arten ist sehr inhomogen. Nur von 10 der 74 an der Wasserlinie gefundenen Spinnenarten wurden mehr als 20 Individuen erfaßt (s. Abb. 2).

Die Baldachinspinne Oedothorax agrestis und die Wolfspinne Pirata knorri sind die mit Abstand am häufigsten vorkommenden Arten.

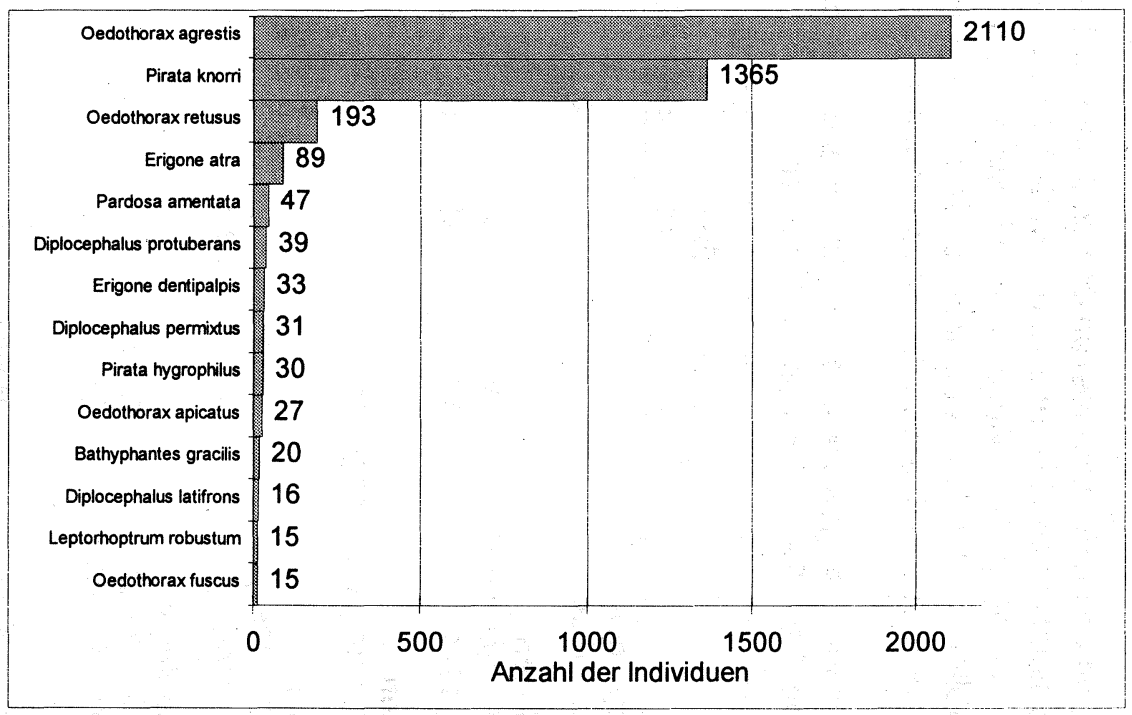

Abb. 2: Individuenanzahl der Spinnenarten, die insgesamt mit 15 oder mehr Individuen an der Wasserlinie nachgewiesen wurden

In den 531 an der Wasserlinie vorgenommenen Handaufsammlungen konnten 74 Spinnenarten und in den 61 in mehr als $1 \mathrm{~m}$ von der Wasserlinie entferntgetätigten Aufsammlungen 29 Spinnenarten nachgewiesen werden. Drei Arten (Enoplognatha ovata, Drepanotylus uncatus, Pityohyphantes phrygianus) kamen ausschließlich in mehr als $1 \mathrm{~m}$ Entfernung von der Wasserlinie vor (s. Tab. 1). 
Tab. 1: Artenliste der echten Spinnen (Araneae) an den acht untersuchten Fließgewässern

LRT: bevorzugter Lebensraumtyp nach Literatur-Angaben (MAURER \& HÄNGGI 1990, HEIMER \& NENTWIG 1991): $U=$ Gewässerufer, $W=$ Wald, $W i=$ Wiese,,$G=$ Gebüsche, Saumgesellschaften, $M=$ Moor, $R=$ Ruderalstandorte, $S=$ Stein-, Geröllfluren, $T=$ Trockenstandorte, $E u$ = überall vorkommend, ? = unsicher

Fundort: $n=$ ufernah, Fundort der Art direkt an der Wasserlinie, $f=$ uferfern, Fundort $>1 \mathrm{~m}$ Entfernung von der Wasserlinie

Gewässer: Acht untersuchte Fließgewässer: $E L=$ Elbrighäuser Bach, $L I=$ Linspher Bach, OR = Orke, $\mathrm{TR}=$ Treisbach, $\mathrm{AL}=\mathrm{Allna}, \mathrm{PE}=$ Perf, $\mathrm{LA}=$ Lahn, $\mathrm{ED}=\mathrm{Eder}$

\begin{tabular}{llllllllllll}
\hline Art & LRT & Fund- & & & Gewässer & & \\
ort & $E L$ & LI & OR & TR AL PE LA ED \\
\hline
\end{tabular}

$\vec{A} \quad$ Agelenidae - Trichternetzspinnen
Histopona torpida (C.L.KOCH, 1834)

W,G $\quad n \quad 2$

Amaurobiidae - Finsterspinnen

Amaurobius fenestralis (STROEM, 1768)

Coelotes terrestris (WIDER, 1834)

$\begin{array}{llllll}W & n & & & 1 & \\ W & n / f & 10 & 2 & & 3\end{array}$

Anyphaenidae - Zartspinnen

Anyphaena accentuata (WALCKENAER, 1802) W

Araneidae - Radnetzspinnen

Larinoides cornutus (CLERCK, 1757)

Mangora acalypha (WALCKENAER, 1802)

Wi,G $\quad n$

$T \quad n / f$

n

1

Gnaphosidae - Plattbauchspinnen

Micaria pulicaria (SUNDEVALL, 1832)

Zelotes subterraneus (C.L.KOCH, 1833)

$M, W$

$\mathrm{Eu}$

n

1

Hahniidae - Bodenspinnen

Antistea elegans (BLACKWALL, 1841)

$\mathrm{M}, \mathrm{Wi},(\mathrm{U}) \quad \mathrm{n}$

1

1 
Linyphiidae - Zwerg- und Baldachinspinnen

Agyneta conigera (O.P.-CAMBR., 1863)

Araeoncus humilis (BLACKWALL, 1841)

Bathyphantes gracilis (BLACKWALL, 1841)

Bathyphantes nigrinus (WESTRING, 1851)

Centromerita bicolor (BLACKWALL, 1833)

Centromerus sylvaticus (BLACKWALL, 1841)

Collinsia distincta (SIMON, 1884)

Dicymbrium nigrum

brevisetosum (LOCKET, 1962)

Dicymbium tibiale (BLACKWALL, 1836)

Diplocephalus cristatus (BLACKWALL, 1833)

Diplocephalus latifrons (O.P.-CAMBR., 1863)

Diplocephalus permixtus (O.P.-CAMBR., 1871)

Diplocephalus picinus (BLACKWALL, 1841)

$\begin{array}{lcccccccc}W, G & \mathrm{n} & 1 & & & & & \\ & \mathrm{n} & & & & 1 & 1 & & 2 \\ \text { W,Wi } & \mathrm{n} & 4 & 6 & & 6 & 1 & 1 & 2 \\ \text { W,Wi } & \mathrm{n} / \mathrm{f} & 4 & & 1 & & & & \\ \text { Eu } & \mathrm{n} & & & 1 & & & & \\ \text { W } & \mathrm{n} / \mathrm{f} & 7 & 2 & 1 & 1 & & 2 & \\ ? & \mathrm{n} & & & & & & & 7\end{array}$

Diplocephalus protuberans (O.P.-CAMBR.,1875)

$\mathrm{M}, \mathrm{Wi}, \mathrm{W} \quad \mathrm{n} / \mathrm{f}$

W

$\begin{array}{lll}5 & 2 & 3\end{array}$

U,Wi

$W$

$W, S,(U)$

$W, G$

Drepanotylus uncatus (O.P.-CAMBR.,1873)

Enteleclara erythropus (WESTRING, 1851)

Erigone atra (BLACKWALL, 1833)

Erigone dentipalpis (WIDER, 1834)

Gonatium paradoxum (L.KOCH, 1869)

Gonatium rubellum (BLACKWALL, 1841)

Gonatium rubens (BLACKWALL, 1833)

Gongylidiellum vivum (O.P.-CAMBR., 1875)

Gongylidium rufipes (LINNAEUS, 1758)

Hilaira excisa (O.P.-CAMBR., 1871)

Lepthyphantes cristatus (MENGE,1866)

Lepthyphantes tenebricola (WIDER, 1834)

Lepthyphantes tenuis (BLACKWALL, 1852)

(U)
W

Eu

Eu

G,W

G,W

G,W

$\mathrm{Eu}$

G

$M, G$

$W$

W

Lepthyphantes zimmermanni BERTKAU, 1890

G

$n / f$
$n$
$n$
$n / f$
$n / f$
$n$
$n / f$
$f$
$n / f$
$n / f$
$n / f$
$n$
$n / f$
$n$
$n$
$n$
$n / f$
$n$
$n / f$
$n / f$
$n / f$

1

1
4

4

$6 \quad 18$

3

22

11

3
2

1

$9 \quad 13$

$\begin{array}{llllll}14 & 5 & 11 & 10 & 14 & 14\end{array}$

$\begin{array}{lll}3 & 1 \\ 5 & 1 & 1\end{array}$

5

5

2




\begin{tabular}{|c|c|c|c|c|c|c|c|c|c|c|}
\hline \multirow[t]{2}{*}{ Art } & \multirow[t]{2}{*}{ LRT } & \multirow{2}{*}{$\begin{array}{l}\text { Fund- } \\
\text { ort }\end{array}$} & \multirow[b]{2}{*}{ EL } & \multicolumn{7}{|c|}{ Gewässer } \\
\hline & & & & $\mathrm{LI}$ & OR & TR & $A L$ & PE & LA & ED \\
\hline Leptorhoptrum robustum (WESTRING, 1851) & $\mathrm{W}, \mathrm{Wi}$ & $\mathrm{n} / \mathrm{f}$ & 7 & 1 & 14 & 1 & & 1 & & \\
\hline Lophomma punctatum (BLACKWALL, 1841) & Wi,M & $\mathbf{n}$ & 1 & & & & & & & \\
\hline Meioneta rurestris (C.L.KOCH, 1836) & $\mathrm{Eu}$ & $\mathrm{n} / \mathrm{f}$ & & 3 & 2 & & & & 4 & 2 \\
\hline Neriene peltata (WIDER, 1834) & W & $n$ & & & & 1 & & & & \\
\hline Oedothorax agrestis (BLACKWALL, 1853) & U, (W), (Wi) & $n / f$ & 905 & 669 & 388 & 273 & 99 & 6 & 41 & 1 \\
\hline Oedothorax apicatus (BLACKWALL, 1850) & $\mathrm{Wi}$ & $\mathrm{n} / \mathrm{f}$ & & 2 & 2 & & 4 & 3 & 13 & 4 \\
\hline Oedothorax fuscus (BLACKWALL, 1834) & Wi,G & $\mathrm{n}$ & 5 & 4 & 3 & 1 & & & 1 & 1 \\
\hline Oedothorax gibbosus (BLACKWALL, 1841) & $M$ & $n$ & 1 & 8 & & & 1 & 1 & & \\
\hline Oedothorax retusus (WESTRING, 1851) & U,Wi & $\mathrm{n} / \mathrm{f}$ & 32 & 48 & 37 & & 2 & & 38 & 48 \\
\hline Pityohyphantes phrygianus (C.L. KOCH, 1836) & W & $f$ & & 1 & & & & & & \\
\hline Porrhomma convexum (WESTRING, 1851) & U,G,(W,Wi) & (i) $n$ & 4 & 2 & & & & & & \\
\hline Porrhomma pygmaeum (BLACKWALL, 1834) & U;W & $n$ & 1 & 1 & 1 & . & & & & \\
\hline Silometopus reussi (THORELL, 1871) & $\mathbf{R}$ & $\mathrm{n}$ & & & & & & & 1 & \\
\hline Tiso vagans (BLACKWALL, 1834) & Wi & $\mathrm{n}$ & 1 & & & & & & & \\
\hline Walckenaeria acuminata BLACKWALL, 1833 & W & $\mathrm{n}$ & 1 & 1 & & & & & & \\
\hline Walckenaeria cuspidata (BLACKWALL, 1833) & W,M & $\mathrm{n}$ & 2 & 1. & & & & & & \\
\hline Walckenaeria nudipalpis (WESTRING, 1851) & Wi,G & $\mathrm{n} / \mathrm{f}$ & 1 & & 1 & & & & & \\
\hline $\begin{array}{l}\text { Liocranidae - Feldspinnen } \\
\text { Agroeca brunnea (BLACKWALL, 1833) }\end{array}$ & Wi,G,W & $\mathrm{n}$ & 1 & & & & & & & \\
\hline $\begin{array}{l}\text { Lycosidae - Wolfspinnen } \\
\text { Alopecosa cuneata (CLERCK, 1757) }\end{array}$ & W & $\mathrm{n}$ & & & & 1 & & & & \\
\hline Pardosa amentata (CLERCK, 1757) & Wi,R,W,U & $\mathrm{n} / \mathrm{f}$ & 3 & 17 & 4 & & & 4 & 20 & 2 \\
\hline Pardosa lugubris (WALCKENAER, 1802) & $W, G$ & $\mathbf{n}$ & 1 & & & & & & & \\
\hline Pardosa palustris (LINNAEUS, 1758 ) & Wi & $\mathrm{n}$ & & & & & & & 1 & \\
\hline Pirata hygrophilus (THORELL, 1872) & W,M,(Wi) & $\mathrm{n} / \mathrm{f}$ & 18 & 1 & 2 & & 2 & 1 & & \\
\hline Pirata knorri (SCOPOLI, 1763) & $\mathrm{U}$ & $\mathrm{n} / \mathrm{f}$ & 59 & 136 & 95 & 18 & & 12 & 16 & 17 \\
\hline
\end{tabular}


Pirata latitans (BLACKWALL, 1833)

Pirata piraticus (CLERCK, 1757)

Trochosa ruricola (DE GEER, 1778)

Trochosa terricola THORELL, 1856

\section{Salticidae - Springspinnen}

Salticus zebraneus (C.L.KOCH, 1837)

Tetragnathidae - Streckerspinnen

Metellina mengei (BLACKWALL, 1869)

Metellina merianae (SCOPOLI, 1763)

Metellina segmentata (CLERCK, 1757)

Pachygnatha clercki SUNDEVALL, 1823

Tetragnatha montana SIMON, 1874

Tetragnatha nigrita LENDL, 1886

Tetragnatha obtusa C.L.KOCH, 1837

\section{$\vec{v} \quad$ Theridiidae - Kugelspinnen}

Enoplognatha ovata (CLERCK, 1757)

Enoplagnatha thoracica (HAHN, 1833)

Theridion varians $\mathrm{HAHN}, 1831$

Thomisidae - Krabbenspinnen

Xysticus cristatus (CLERCK, 1757)

$\begin{array}{ll}W i,(U) & n \\ U & n \\ W i & n \\ W, G, T & n\end{array}$

?

n

$W, G$

$G,(U)$

$W, G$

Wi

G,(U)

G,(U)

G

$\begin{array}{ll}G & f \\ T & n \\ W, G & n\end{array}$

$\mathrm{Eu}$

n 1 
Die drei Baldachinspinnen Erigone atra, Erigone dentipalpis und Oedothorax agrestis kommen an allen acht Fließgewässern vor (s. Tab. 1). Neben Oedothorax agrestis und Pirata knorri haben Diplocephalus permixtus und Diplocephalus protuberans ihren Verbreitungsschwerpunkt an den Mittelgebirgsbächen, wobei die letztgenannte Art vor allem im Epirhithralbereich zu finden ist. Im Gegensatz zu Oedothorax agrestis hat Oedothorax retusus ihren Vorkommensschwerpunkt an Lahn und Eder. Neben dieser Art sind noch Oedothorax apicatus, Erigone atra und Pardosa amentata auf fast allen Untersuchungsabschnitten von Lahn und Eder nachgewiesen worden. Pirata knorri tritt gleichermaßen an den Mittelgebirgsbächen wie an Lahn und Eder auf.

Fünf Spinnenarten sind in der Roten Liste Deutschlands (PLATEN et al. 1996) vertreten. Danach gehört die Wolfsspinne Pirata knorri den stark gefährdeten Arten an, die Baldachinspinnen Collinsia distincta, Drepanotylus uncatus und Gonatium paradoxum gehören zu den gefährdeten Arten und eine weitere Baldachinspinne (Diplocephalus protuberans) wird als Art eingestuft, deren geographische Verbreitung begrenzt ist.

\section{Abundanz der Spinnen an den Untersuchungsabschnitten}

Die Abundanzen in den einzelnen Proben schwanken über zwei Größenordnungen. Zur Charakterisierung der Individuendichten an den Untersuchungsabschnitten wird daher der Median der in allen Proben festgestellten Dichten verwendet.

Am Elbrighäuser Bach konnten die höchsten mittleren Individuendichten von 15-58 Ind. $/ \mathrm{m}^{2}$ nachgewiesen werden. Die Abundanz nimmt hier mit wachsender Entfernung von der Quelle deutlich zu (s. Abb. 3).

An den übrigen Gewässern, an denen sich immer mindestens ein stark verbauter Abschnitt befand, konnte solch ein Trend nicht beobachtet werden. Die mittleren Abundanzen (Mediane) liegen hier zwischen 4 und 28 Individuen $/ \mathrm{m}^{2}$.

Die höchsten Individuendichten wurden auf Schotterbänken des Linspher Baches (LI2, LI3) und des Elbrighäuser Baches (EL3) vorgefunden. Auf bis zu $4 \mathrm{~m}^{2}$ großen Schotterbänken wurden 180 (32\% Adulte) bzw. 200 (68\% Adulte) Individuen $/ \mathrm{m}^{2}$ aufgesammelt.

Aufgrund der insgesamt sehr großen Abundanzschwankungen kann kein Zusammenhang zwischen dem Verbauungsgrad der Gewässerabschnitte und den mittleren Abundanzen hergestellt werden. 


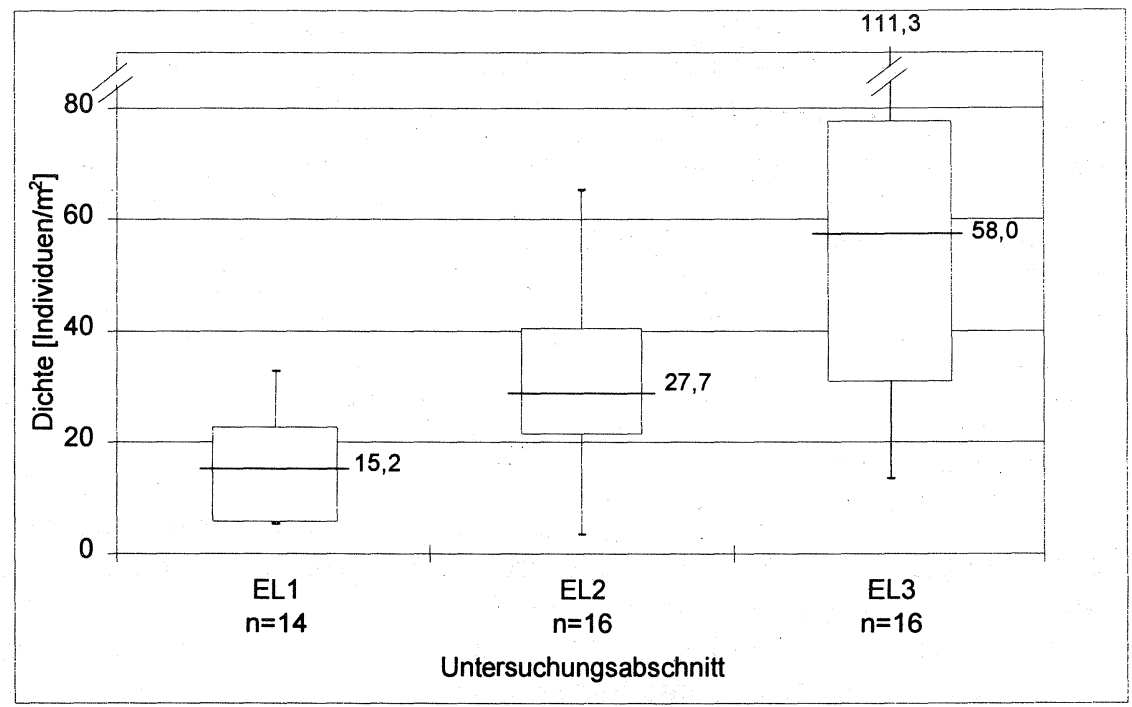

Abb. 3: Individuendichte (Individuen $/ \mathrm{m}^{2}$ ) der echten Spinnen (Araneae) an den drei Untersuchungsabschnitten des Elbrighäuser (dargestellt in "Boxand-Whisker-Plots" (Spannbreite, 1. und 3. Quartil, Median, n= Anzahl der untersuchten Schotterbänke)

\section{Abundanzen einzelner Spinnenarten}

Die dominierenden Arten erreichten maximale Abundanzen zwischen 91 und $121 \mathrm{Ind} . / \mathrm{m}^{2}$ (s. Tab. 2).

Oedothorax agrestis trat auf einer voll beschatteten Schotterbank am Elbrighäuser Bach (EL3) im dritten Arbeitsdurchgang (September) mit der höchsten Individuendichte (114,3 Ind./ $\mathrm{m}^{2}$ ) auf (s. Tab. 2).

Oedothorax retusus kommtgrundsätzlich in wesentlich geringerer Dichte als ihre Schwesterart vor (s. Tab. 2). Ihre maximale Abundanz erreichte sie an derEder (ED2) im ersten Arbeitsdurchgang (Mai) auf einervoll besonnten Schotterbank.

Pirata knorri trat mit maximaler Individuendichte auf einer Schotterbank am Linspher Bach (LI3) im zweiten Durchgang (Juli) auf (s. Tab. 2). Allerdings lag der Anteil juveniler Individuen bei 58,8\%. 
Tab. 2: Die maximal in einer Handaufsammlung festgestellten Individuendichten von Oedothorax agrestis, Oedothorax retusus und Pirata knorri unter Angabe der Schotterbankgröße und des Gewässers

\begin{tabular}{lccccccc}
\hline Art & Datum Schotterbank- & Gewässer & \multicolumn{3}{c}{ Dichte [Individuen/m ${ }^{2}$ ] } \\
& & größe & & Gesamt & Adulte & Juvenile Subad. \\
\hline O. agrestis & 03.10 .95 & $2,5 \mathrm{~m}^{2}$ & Elbrighäuser B. & 114,3 & 114,3 & - & - \\
O. retusus & 24.07 .95 & $15 \mathrm{~m}^{2}$ & Eder & 26,8 & 26,8 & - & - \\
\hline P. knorri & 26.07 .95 & $4 \mathrm{~m}^{2}$ & Linspher B. & 121,4 & 50 & 71,4 & 0 \\
P. knorri & 25.09 .95 & $3,5 \mathrm{~m}^{2}$ & Linspher B. & 91 & 58,9 & 23,2 & 8,9 \\
\hline
\end{tabular}

\section{Ökologische Typen}

Der prozentuale Anteil derUferarten istan allen untersuchten Fließgewässern sehrgering (s. Abb. 4). Vielmehr finden sich an den Gewässern Spinnenarten aus ganz verschiedenen ökologischen Gruppen.

Die prozentuale Verteilung der Individuen auf die ökologischen Typen zeigt jedoch ein ganz anderes Bild (s. Abb. 5). Über $90 \%$ der Individuen gehören an den naturnahen Gewässern zu den Uferarten. An diesen Gewässern sind die eurytopen Spinnenarten deutlich weniger stark vertreten als an den übrigen Fließgewässern. An wasserbaulich veränderten Flüssen wie Allna, Lahn und Eder sind weniger als $80 \%$ der erfaßten Spinnen ripicolen Arten zuzuordnen, an der Perf sogar weniger als $50 \%$.

Pirata knorriund Oedothorax agrestis, die im wesentlichen die Uferarten repräsentieren, zeigen eine sehr unterschiedliche Verteilung auf die Untersuchungsabschnitte. $O$. agrestis kommt überall, unabhängig vom Verbauungsgrad und der Quellentfernung der Schotterbank vor. P. knorri hingegen tritt verstärkt an den besonnten und naturnahen Gewässerabschnitten auf. Sie fehlt fast vollständig an stark verbauten Abschnitten, an denen Schotterbänke nur vereinzelt auftreten.

\section{DISKUSSION}

\section{Die Spinnenzönose an Mittelgebirgsbächen}

Auch wenn die Spinnenzönose auf Schotterbänken der Mittelgebirgsbäche im wesentlichen von nur zwei Arten (Oedothorax agrestis, Pirata knorri) geprägt wird, kann man trotzdem von einer eigenständigen, charakteristischen Uferfauna sprechen. Das Umland scheint zwar einen Einfluß auf die Artenzusammensetzung auf den Schotterbänken auszuüben, jedoch zeigen die niedrigen Individuenanteile der eingewanderten Arten, daß sie sich nicht auf den Schotterbänken etablieren können. 


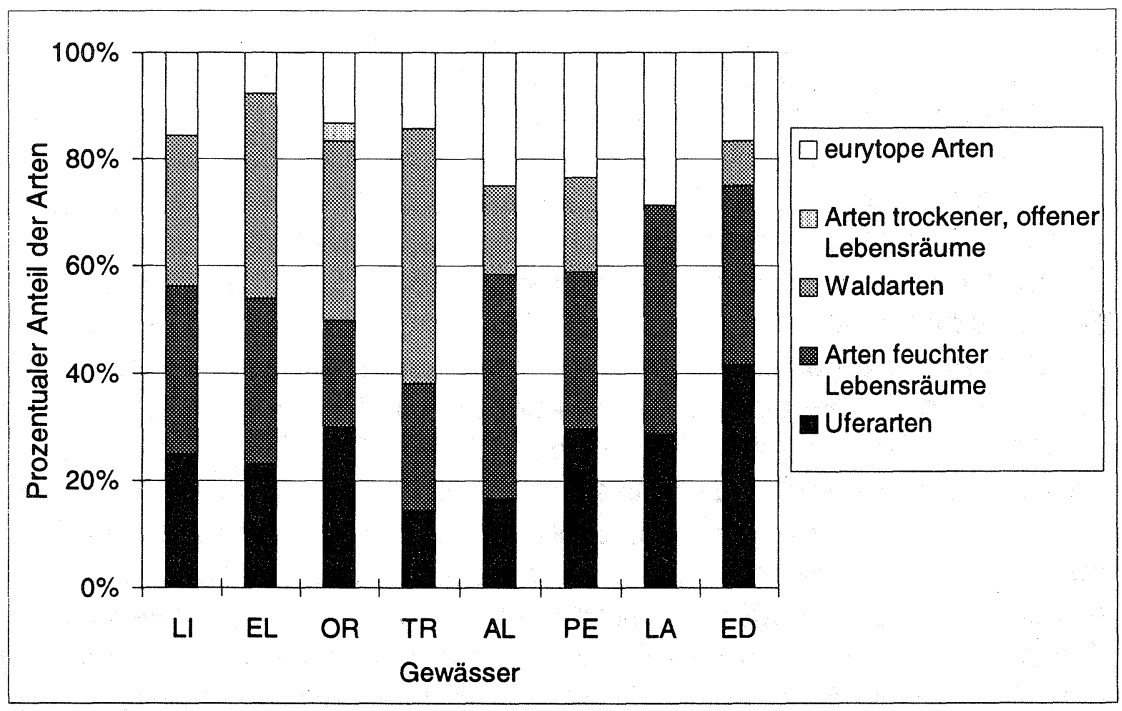

Abb. 4: Prozentualer Anteil derSpinnenarten an den ökologischen Typen (Einordnung nach MAURER \& HÄNGGI 1990)

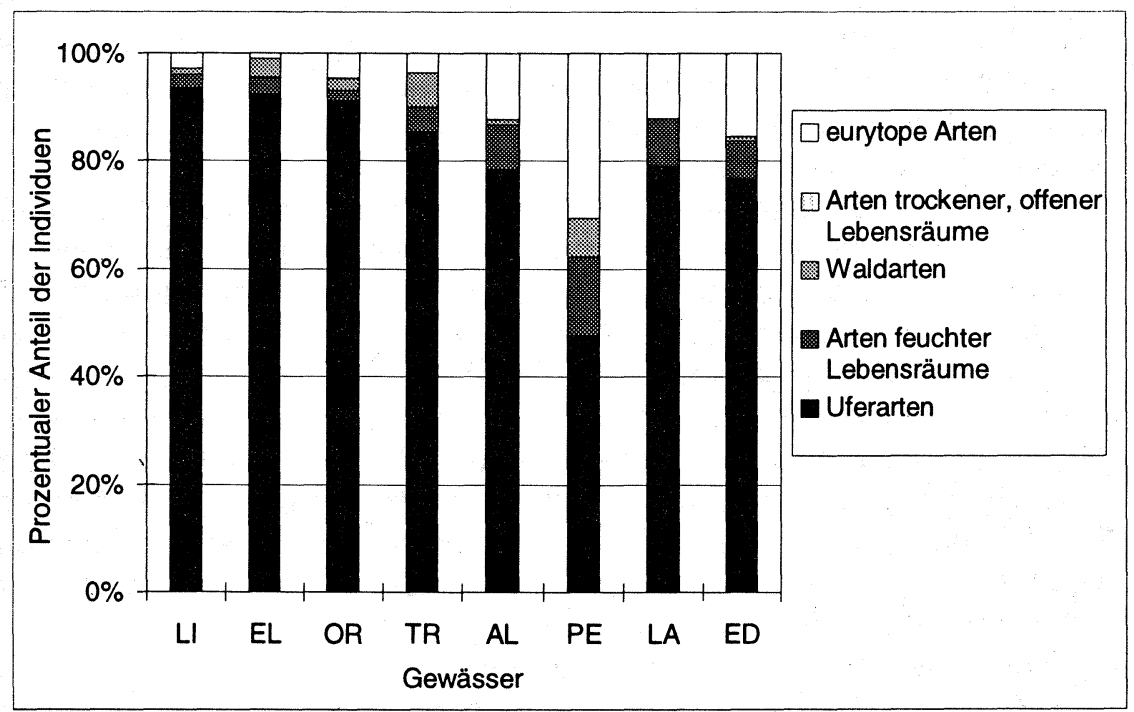

Abb. 5: Prozentualer Anteil der Individuen an den ökologischen Typen (Einordnung nach MAURER \& HÄNGGI 1990) 
Der Uferbereich der Schotterflächen an Mittelgebirgsbächen im Schiefergebirge ist von starken Wasserstandsschwankungen geprägt. Die häufig überfluteten und wieder trockenfallenden Schotterbänke stellen somit einen extremen Lebensraum dar. Wie die hier vorliegenden Ergebnisse zeigen, bedingtdiese Kurzlebigkeit des Lebensraumes, daß nurwenige Spinnenarten sich dort „auf Dauer" etablieren können. Nicht ein großes Spektrum an Spinnenarten ist hier zu finden, sondern wenige Spezialisten bilden die Spinnenzönose der Uferbereiche.

Oedothorax agrestis und Pirata knorri sind Charakterarten von Schotterufern. Neu gebildete Schotterbänke könnten von Oedothorax agrestis, die zu den sogenannten Aeronauten gehört (SCHÄFER 1976), über den Luftweg schnell besiedelt werden. Dies ist sicherlich ein entscheidender Faktor, da selbst im Sommer die Uferbereiche der Schotterflächen innerhalb kürzester Zeit überwemmt werden und wieder trockenfallen. Selbst die isoliert liegenden Schotterbänke in den stark verbauten Abschnitten könnte Oedothorax agrestis auf diese Weise erreichthaben, da eine Einwanderung aus dem angrenzenden Umland weitgehend ausgeschlossen werden kann (s.u.). In diesen stark verbauten Abschnitten ist Pirata knorri kaum anzutreffen. Sie gehört im Gegensatz zu Oedothorax agrestis nicht zu den Arten, die sich regelmäßig als Aeronauten über den Luftweg verbreiten. Allenfalls im juvenilen Zustand könnte ein Fliegen am Fadenfloß möglich sein (RICHTER 1970). Ansonsten ist Pirata knorri aufgrund ihres Gewichtes höchstwahrscheinlich auf eine Ausbreitung auf dem Landweg oder eventuell über die Wasseroberfläche angewiesen. Isoliert liegende Schotterbänke sind so schwerer zu besiedeln.

Nach den hier vorliegenden Ergebnissen ist davon auszugehen, daß Pirata knorri regelmäßig auf den Schotterflächen naturnaherFließgewässer im Rheinischen Schiefergebirge anzutreffen ist. Vorkommen dieser Art sind jedoch auch an schotterbildenden Mittelgebirgsgewässern außerhalb des Rheinischen Schiefergebirges zu vermuten (s. DAHL 1908). So konnte die Art 1993 mit einer individuenreichen Population auf einer Schotterbank an der IIm (Thüringen) nachgewiesen werden (MALT, mdl. Mitteilung). Das weitgehende Fehlen aktueller Nachweise von Pirata knorri im außeralpinen Bereich dürfte weniger auf das Fehlen dieser Art im ebengenannten Lebensraum zurückzuführen sein, als vielmehr die Unerforschtheit der schotterführenden Mittelgebirgsbäche widerspiegeln.

Die Tatsache, daß Schotterbänke nur noch an naturnahen Bächen in größerer Anzahl zu finden sind, da Verbauungsmaßnahmen zu einem extremen Rückgang dieses Lebensraumtyps führen (SMIT et al. i. Dr.), zeigt den hohen Gefährdungsgrad dieses Biotoptyps auf. Stenöke 
schotterbewohnende Uferarten, wie zum Beispiel Pirata knorri, die zudem nur beschränkte Ausbreitungsmöglichkeiten besitzt, sind bei Verlust dieses Lebensraumtyps hochgradig gefährdet.

Im folgenden werden Verbreitung und Habitatansprüche ausgewählter Spinnenarten diskutiert.

Pirata knorri (SCOPOLI, 1763)

Die Besonnung scheint für die Verteilung von Pirata knorri ein mitbestimmender Standortfaktor zu sein. So konnten besonders hohe Dichten an den im fast durchgängig besonnten Wiesental gelegenen Abschnitten des Linspher Baches (LI2, LI3) nachgewiesen werden. Außerdem war an sonnigen Tagen eine deutlich höhere Laufaktivität von Pirata knorri auf den Schotterbänken zu beobachten. Pirata knorri lebt am Geröllufer der Bäche und Flüsse (HEIMER \& NENTWIG 1991, MAURER \& HÄNGGI 1990, DAHL \& DAHL 1927). Im Gegensatz zu anderen Pirata-Arten ist Pirata knorri bisher nur an Fließgewässern, nicht aber an stehenden Gewässern oder in anderen feuchten Lebensräumen beobachtet worden (vgl. RENNER 1986).

An alpinen Fließgewässern ist Pirata knorri weit verbreitet (FRAMENAU et al. 1996, HERING 1995, PLACHTER 1986). Sobald der Geröllstreifen breiter ausgeprägt ist, wird sie dort jedoch von Pardosa wagleri ersetzt (DAHL 1908, vgl. DRÖSCHMEISTER 1994).

Im außeralpinen Bereich sind nur sehr vereinzelt Fundorte dieser Art bekannt. JÄGER \& KREUELS (1995) fanden sie am Schotterufer eines Baches im Bergischen Land. DAHL (1908) wies sie an Geröllufern im Thüringer Wald, im Harz, im Frankenwald und im Böhmerwald nach. Trotz derwenigen aktuellen Nachweise dieser Art, kann nach den hier vorliegenden Ergebnissen davon ausgegangen werden, daß Pirata knorri auf Schotterflächen der Mittelgebirge regelmäßig auftritt. Allein die Unerforschtheit dieses Lebensraumes dürfte der Grund für die nur wenigen Nachweise im außeralpinen Raum sein.

Oedothorax agrestis (BLACKWALL, 1853)

Bisher konnte Oedothorax agrestis überwiegend an steinigen Ufern nachgewiesen werden (PALMGREN 1976, WIEHLE 1960, MAURER \& HÄNGGI 1990). Lediglich MÜLLER(1983) und NENTWIG (1983) berichten über das Vorkommen weniger Exemplare dieser Art an sumpfigen Stellen. DROSCHMEISTER (1994), FRAMENAU et al. (1996), HERING (1995), PLACHTER (1986) und STEINBERGER(i. Dr.) wiesen Oedothorax agrestis auf Schotterbänken alpiner und dealpiner Fließgewässer nach. MARTIN (1983) fand sie am strukturarmen „Ostufer derMüritz" (NSG) in MecklenburgVorpommern. 
Oedothorax retusus (WESTRING, 1851)

Anders als ihre Schwesterart Oedothorax agrestis verhält sich Oedothorax retusus, die inren Verbreitungsschwerpunkt im Untersuchungsgebiet eindeutig an den etwas größeren Fließgewässern Lahn und Eder hat.

CASEMIR (1962) bezeichnet Oedothorax retusus als auffällige Charakterart der gut belichteten, grundwassernahen Grünlandufer. Im Gegensatz zu Oedothorax agrestis scheint sie nicht ausschließlich an Gewässerufern (vgl. z. B. DRÖSCHMEISTER 1994, FRAMENAU et al. 1996, JOOST 1991, PLACHTER 1986) aufzutreten, sondern ist regelmäßig auch in anderen feuchten Lebensräumen, wie in Mooren (ANDREESSEN 1993, PLATEN 1989) und an der Nord- und Ostseeküste (HEYDEMANN 1961, SCHÄFER 1971) anzutreffen.

\section{Diplocephalus permixtus (O.P.-CAMBRIDGE, 1871)}

Diplocephalus permixtus, die im Rahmen der vorliegenden Untersuchung in geringen Individuendichten ausschließlich an beschatteten Abschnitten der Mittelgebirgsbäche auftrat, wird als stenök-hygrophil bezeichnet(BRAUN 1961, HEIMER \& NENTWIG 1991, MAURER \& HÄNGGI 1990, WIEHLE 1960). SCHENKEL (nach WIEHLE 1960) stuftsie als Leitform für Quellgebiete ein. Sie kommt sowohl an der Küste (SUDD 1972) als auch an Ufern von Gewässern und in Mooren vor (z.B. ANDREESSEN 1993, ASSMUTH 1981, CASEMIR 1962, DRÖSCHMEISTER 1994, JOOST 1991, KNÜLLE 1952, MARTIN 1983, PLATEN 1989,1994). Nachweise aus Hessen liegen von ASSMUTH (1981) und MÜLLER (1983) vor.

Diplocephalus protuberans (O.P.-CAMBRIDGE, 1875)

Diplocephalus protuberans, die im Rahmen dieser Untersuchung schwerpunktmäßig in den Epirhithralbereichen der Bäche nachgewiesen wurde, scheint ebenfalls eng an feuchte Lebensräumegebunden zu sein. ROBERTS (1975) fand sie an einem Bach in North Wales. THALER (1978) konnte sie in Nord- und Südtirol an einem Bachgraben und einem Quellsumpf nachweisen. Ansonsten liegen noch Nachweise von DENIS (1947) und CASEMIR (1962) vor. Die Art wurde in Hessen bislang noch nicht gefunden (MALTEN mdl. Mitt.).

Collinsia distincta (SIMON, 1884)

In den vorliegenden Untersuchungen konnte diese Art lediglich auf Schotterbänken an der Lahn nachgewiesen werden. Von dieser Art liegen bisher wenig Nachweise aus Mitteleuropa vor, sie wird als selten bezeichnet (HEIMER \& NENTWIG 1991, WIEHLE 1960). CASEMIR (1962) bezeichnet sie als zönobionte Art des Glyceria-Röhrichts. Ansonsten liegen Funde von der Nord-und Ostseeküste vor (WIEHLE 1960). Außerdem wurde Collinsia 
distincta auf Schotterbänken an der Isar (PLACHTER 1986) und in den östlichen Donauauen gefunden (THALER et al. 1984).

Hilaira excisa (O.P.-CAMBRIDGE, 1871)

Diese Art wurde an zwei naturnahen Metarhithralabschnitten gefunden. Bisherwurde die Artzumeist in Torfmoos gefunden (s. BRAUN 1961). Auch WIEHLE (1956) bezeichnet sie als sphagnobionte Art. ASSMUTH (1981) vermutet, daß die Verbreitung dieser Art auf Hochmoore beschränkt ist. JOOST (1991) jedoch fand sie ebenfalls an einem Mittelgebirgsbach (in Emergenzfallen, Thüringer Wald).

\section{Silometopus reussi (THORELL, 1871)}

Diese Art wurde ausschließlich auf Schotterbänken an der Lahn gefunden. HEYDEMANN (1962) wies diese Art im Jungkoog nach. MARTIN (1983) fand Silometopus reussi in nassen bis feuchten Substraten im offenen Gelände im Naturschutzgebiet „Ostufer der Müritz".

\section{ZUSAMMENFASSUNG}

Von Mai bis Oktober 1995 wurden am östlichen Rand des Schiefergebirges Untersuchungen zur epigäischen Spinnenfauna auf Schotterbänken an Fließgewässern durchgeführt. Die Untersuchung erstreckte sich auf acht Fließgewässer: vier kleinere in weiten Strecken naturnahe Gewässer, zwei kleinere, stark verbaute Gewässer und zwei größere Fließgewässer im Epipotamalbereich. An den kleineren Gewässern wurden jeweils ein Kilometer lange Abschnitte im Epi-, Meta- und Hyporhithralbereich, an den größereren Gewässern wurden mehrere ein Kilometer lange Abschnitte bearbeitet. Die Erfassung der Spinnenfauna erfolgte in drei Arbeitsdurchgängen (Mai, Juli, September) durch insgesamt 592 flächenbezogene Handaufsammlungen.

Es wurden insgesamt 7027 Spinnen (Araneae) aus 77 Arten gefunden. Oedothorax agrestis und Pirata knorri stellen $72 \%$ der adulten Spinnen. Darüberhinaus traten neben Erigone atra und Erigone dentipalpis an den größeren Gewässern noch Oedothorax retusus und Pardosa amentata, an den Bächen noch Diplocephalus protuberans und Diplocephalus permixtus in höheren Abundanzen auf. Diplocephalus protuberans wurde erstmals für Hessen nachgewiesen. Die mittlere Abundanz (Median) aller Spinnen schwankte an den Untersuchungsabschnitten zwischen 4 und $28 \mathrm{Ind} . / \mathrm{m}^{2}$. Die maximal registrierte Individuendichte betrug 198 Spinnen $/ \mathrm{m}^{2}$. 
Danksagung und Fördernachweis: Herrn Dr. K. THALER danke ich für die Bestätigung der Bestimmung von Diplocephalus protuberans. Herr Dipl. Biol. V.FRAMENAU (Marburg/ Melbourne) übernahm die Nachbestimmung einiger kritischer Spinnenarten. Für die genaue Fundortangabe von Pirata knorri möchte ich Herrn Dipl.-Biol. P.JÄGER (Mainz) danken. Herrn Dr. D.HERING (Marburg) und Herrn Dr. M.REICH (Marburg) danke ich für die kritische Durchsicht des Manuskriptes. Die Untersuchungen wurden als Teil des Vorhabens "Ökologie und Schutz alpiner Wildflüsse" (Fördernummer 0339530 ) vom Bundesministerium für Bildung, Wissenschaft, Forschung und Technologie (BMBF) gefördert.

\section{LITERATUR}

ALMQUIST, S. (1969): Seasonal growth of some dune-living spiders. - Oikos 20: 392-408 ALMQUIST, S. (1973): Spider association in costal sand dunes. - Oikos 24: 444-457

ANDREESSEN, B. (1993): Spinnen (Araneida) in Mosaikbiotopen eines degenerierten Hochmoorrestes in Niedersachsen. - TELMA 23: 181-198 (Hannover)

ASSMUTH (1981): Zur Spinnenfauna (Arachnida, Araneae) des Naturdenkmals "Weiberhemdmoor" am Hohen Meißner. - Hess. faun. Briefe 1: 60-69 (Darmstadt)

BIGOT, L. \& C.FAVET (1985): La caumunauté ripicole des araignées de la Basse Durance. - Bull. Soc. Linn. Provence 37: 53-67

BIGOT, L. \& G.GAUTIER (1982): La comunauté des arthropodes des rives de l'Ouvèze (Vaucluse). - Ecologia mediterranes 8(4): 11-36

BOUMEZZOUGH, A. \& J.J.MUSSO (1983): Etude des communautés animales ripicoles du bassin de la rivière Aille (Var-France). I: Aspects biologiques et éco-éthologique. - Ecologia Mediterranes 9(1): 31-56

BOUMEZZOUGH, A. (1983): Les communautés animales ripicoles du bassin versant de la rivière Aille (Var-France). II: Composition et structure de la faune épigée. - Ecologia Mediterranes 9(2): 3-33

BRAUN, R. (1961): Zur Kenntnis der Spinnenfauna an Fichtenwäldern höherer Lagen des Harzes. - Senck. biol. 42(4): 375-395

CASEMIR, H. (1962): Spinnen vom Ufer des Altrheins bei Xanten/Niederrhein. - Gewässer und Abwässer 30/31: 7-35

DAHL, F. (1908): Die Lycosiden oder Wolfsspinnen Deutschlands und ihre Stellung im Haushalte der Natur. - Nova Acta Leopoldina, Abh. Leop.-Carol. Dtsch. Akad. der Naturforscher 88: 175-678

DAHL, F. \& M.DAHL (1927): Spinnentiere oder Arachnoidea: II: Lycosidae s. lat. (Wolfsspinnen im weiteren Sinne). - F. Dahl (Hrsg.): Die Tienwelt Deutschlands und der angrenzenden Meeresteile nach ihren Merkmalen und ihrer Lebensweise, 5. Teil, Gustav Fischer Verlag, Jena; $80 \mathrm{~S}$.

DENIS, J. (1947): Notes sur les Erigonides: XI. Les espèces francaises du genre Oedothorax Bertkau. - Bull. Soc. Hist. nat. Toulouse 82: 131-158

DRÖSCHMEISTER, R. (1994): Die Spinnenfauna der Kies- und Schotterbänke des nordalpinen Wildbaches Halblech (Landkreis Ostallgäu). - Ber. Naturwiss. Ver. Schwaben 98(3): $61-70$

FRAMENAU, V., M.REICH \& H.PLACHTER (1996): Zum Wanderverhalten und zur Nahrungsökologie von Arctosa cinerea (Fabricius, 1777) (Araneae: Lycosidae) in einer alpinen Wildflußlandschaft. - Verh. Ges. Ökol. 26: 369-376 
HEIMER, S. \& W.NENTWIG (1991): Spinnen Mitteleuropas. - Verlag Paul Parey; Berlin, 543 S. HERING, D. (1995): Nahrungsökologische Beziehungen zwischen limnischen und terrestrischen Zoozönosen im Uferbereich nordalpiner Fließgewässer. - Dissertation am Fachbereich Biologie, Marburg; 207 S.

HEYDEMANN, B. (1961): Verlauf und Abhängigkeit von Spinnensukzession im Neuland der Nordseeküste. - Verh. Dt. Zool. Ges. 24: 431-519

JÄGER, P. \& M.KREUELS (1995): Liste der Spinnen (Araneae) von Nordrhein-Westfalen. - Mitt. ArbGem. ostwestf.-lipp. Ent. 11 (Beiheft 2): 1-3

JOOST, W. (1991): Araneae aus der Sicht der Spitter-Emergenzfalle im Thüringer Wald als Beitrag zur Kenntnis der Uferfauna eines Mittelgebirgbaches. - Entomol. Nachrichten Ber. 35(1): 17-27

KNÜLLE, W. (1953): Zur Ökologie der Spinnen an Ufern und Küsten. - Z. Morph. u. Ökol. Tiere 42: $117-158$

MANDERBACH, R. \& M.REICH (1995): Auswirkungen großer Querbauwerke auf die Laufkäferzönosen (Coleoptea, Carabidae) von Umlagerungsstrecken der Oberen Isar. - Arch. Hydrobiol. Suppl. (Large Rivers 9)(3/4): 573-588

MARTIN, D. (1983): Die Spinnenfauna des Naturschutzgebietes „Ostufer der Müritz". - Zool. Rundbf. Bez. Neubrandenburg 3: 1-40

MAURER, R. \& A.HÄNGGI (1990): Katalog der Schweizerischen Spinnen. - Documenta Faunistica Helvetica 12, Neuchâtel, $412 \mathrm{~S}$.

MÜLLER, H.-G. (1983): Zum Vorkommen von Oedothorax agrestis (BLACKWALL) (Araneida, Linyphiidae) in Hessen. - Hess. faun. Briefe 3(4): 64-67

NENTWIG, W. (1983): Die Spinnenfauna (Araneae) eines Niedermoores (Schweinsberger Moor bei Marburg). - Decheniana 136: 43-51

PALMGREN, P. (1976): Die Spinnenfauna Finnlands und Ostfennoskandinaviens VII, Linyphiidae 2 (Micryphantinae, mit Ausnahme der Linyphiinae-Ähnlichen). - Fauna Fennica 29: 1-126

PLACHTER, H. (1986): Die Fauna der Kies- und Schotterbänke dealpiner Flüsse und Empfehlungen für ihren Schutz. - Ber. ANL 10: 119-147

PLACHTER, H. (1993): Alpine Wildflüsse. - Garten Landschaft 4: 47-52

PLATEN, R. (1989): Struktur der Spinnen- und Laufkäferfauna (Arach.: Araneida, Col.: Carabidae) anthropogen beeinflußter Moorstandorte in Berlin (West); taxonomische, räumliche und zeitliche Aspekte. - Diss. TU Berlin; $470 \mathrm{~S}$.

PLATEN, R. (1994): Räumliche und zeitliche Verteilung der Spinnentier- (Arach.: Araneida, Opilionida) und Laufkäferfauna (Col.: Carabidae) im NSG Radauer Born. - Hercynia (Halle) 29: 57-100

PLATEN, R., T.BLICK, P.SACHER \& A.MALTEN (1996): Rote Liste der Webspinnen Deutschlands (Arachnida: Araneae). - Arachnol. Mitt. 11: 5-31

PLATNICK, N.I. (1993): Advances in Spider Taxonomy 1988-1991. With synonymies and transfers 1940-1980. - New York Entomological Society \& The American Museum of Natural History, New York; 846 S.

RENNER, F. (1986): Zur Nischendifferenzierung bei Pirata-Arten (Araneida, Lycosidae). - Verh. naturwiss. Ver. Hamburg 28: 75-90

RICHTER, D.J.J. (1970): Aerial dispersal in relation to habitat in eight wolf spider species (Pardosa, Araneae, Lycosidae). - Oecologia 5: 200-214

ROBERTS, M. J. (1975): A note on the female of Diplocephalus protuberans. - Bull. Br. arachnol. Soc. 3 (5): 144 
ROBERTS, M.J. (1993a): The spiders of Great Britain and Ireland Volume 1: Atypidae to Theridiosomatidae. - Harley Books, Martins; 229 S.

ROBERTS, M.J. (1993b): The spiders of Great Britain and Ireland Volume 2: Linyphiidae and Checklist. - Harley Books, Martins; 217 S.

ROBERTS, M.J. (1993c): The spiders of Great Britain and Ireland Volume 3: Plates. - Harley Books, Martins; $230 \mathrm{~S}$.

RUZICKA, V. (1978): Revision der diagnostischen Merkmale der Weibchen der tschechoslowakischen Arten der Gattung Oedothorax (Araneae: Micryphantidae). - Vestnik Ceskoslovenske Spolecnosti Zool. 42: 195-208

SCHÄFER, M. (1971): Zur Jahresperiodizität der Spinnenfauna einer Ostseeküstenlandschaft. - Biol. Zbl. 90: 579-609

SCHÄFER, M. (1976): Experimentelle Untersuchungen zum Jahreszyklus und zur Überwinterung von Spinnen. - Zool. Jb. Syst. 108: 127-289

SIEPE, A. (1985): Einfluß häufiger Überflutungen auf die Spinnen-Besiedlung am OberrrheinUfer. - Mitt. dt. Ges. allg. angew. Ent. 4: 281-284; Kiel

SMIT, J., J.HOPPNER, D.HERING \& H.PLACHTER (i. Dr.): Struktur, Spinnen- und Laufkäferfauna (Araneae, Carabidae) von Kiesbänken an Mittelgebirgsbächen Nordhessens. - Verh. Ges. Ökol. 27

STEINBERGER, K.H. (i. Dr.): Die Spinnenfauna der Uferlebensräume des Lechs (Arachnida: Araneae) (Nordtirol, Österreich). - Ber. nat.-med. Ver. Innsbruck 83

SUDD, J. H. (1972): The distribution of spiders at Spurn Head (E. Yorkshire) in relation to flooding. - J. Animal Ecol. 41: 63-70

THALER, K. (1978): Über wenig bekannte Zwergspinnen aus den Alpen - V (Arachnida: Aranei, Erigonidae). - Beitr. Ent., Berlin 28(1): 183-200

THALER, K., M.PINTAR \& H.M.STEINER (1984): Fallenfänge von Spinnen in den östlichen Donauauen (Stockerau, Niederösterreich). - Spixiana 7(2): 97-103

WIEHLE, H. (1960): Spinnentiere oder Arachnoidea (Araneae): XI. Micryphantidae - Zwergspinnen. - DAHL, F. (Begründer): Die Tienwelt Deutschlands, 47. Teil. G. Fischer Verlag, Jena; $620 \mathrm{~S}$.

Janna SMIT, Phillips-Universität Marburg, Fachbereich Biologie - Zoologie Abt. Tierökologie, Karl-von-Frisch-Str., D-35032 Marburg 